Backround: Iron deficiency anemia (IDA) and thalassemia are the most common microcytic anemia in children. Sometimes, expensive or invasive investigations are necessary for their distinction. Red cell distribution - coefficient variation (RDW-CV) is a new generation marker more often used in differentiating IDA from thalassemia. A number of hematological indices are also useful (Mentzer, Shine\&Lal, Green\&King, Ricerca).

Aim To demonstrate the effectiveness RDW - CV in differentiating microcytic anemia.

Method Retrospective study conducted on a total of 215 patients with microcytic anemia. Criteria for study group selection: MCV and $\mathrm{Hb}$ below the minimum age reference (mild anemia, $\mathrm{Hb}$ between 9-11 g/dl and moderate anemia from 8.5-9 g/dl), without a history of other hematologic disorders or chronic diseases.

Sideremia was determined to all patients to confirm the diagnosis of IDA (the least expensive investigation for distinction). The RDW - CV $>16 \%$ was considered pathological.

Results 98 patients had low levels of sideremia, 134 patients had mild anemia and 81 - moderate anemia. RDW-CV was significantly correlated with sideremia, strongly for the group with mild anemia $(p<0.05)$. Statistical significance was observed for Shine\&Lal $(p<0.006)$, Green \& King $(p<0.005)$, Ricerca $(p<0.05)$. The greatest sensitivity presented Green\&King (72\%), followed by Shine\&Lal and Ricerca, and the highest specificity Shine\&Lal and Ricerca (92\%).

Conclusions RDW-CV is useful in differentiating IDA, especially in mild forms. Although Green\&King index is the most sensitive, we support Ricerca index for simple calculation formula.

\section{PREVALENCE OF MATERNAL ANEMIA AND ITS ASSOCIATION WITH HEMOGLOBIN LEVELS OF NEWBORN BABIES}

doi:10.1136/archdischild-2012-302724.0757

${ }^{1} \mathrm{~N}$ Esmailnasab, ${ }^{1} \mathrm{~A}$ Afkhamzadeh, ${ }^{2} \mathrm{~A}$ Delpisheh. ${ }^{1}$ Kurdistan University of Medical Sciences, Sanandaj; 21 lam University of Medical Sciences, Ilam, Iran

Background and Aims There are some reports regarding to the association between maternal and fetal hemoglobin level. The present study aimed to determine the prevalence of maternal anemia and its association with hemoglobin levels in their newborn babies. Methods It was a descriptive analytical study in which mothers who referred for delivery to the Besat Hospital in Sanandaj city, Kurdistan province, western Iran were investigated. Overall, 604 mothers was recruited using easy sampling method. Blood assessment was conducted using the cell counter machine. The statistical tests of Chi square and $t$ were used. Meanwhile, the Spearman correlation coefficient was used to test all correlations. The multiple regression analysis was also undertaken to assess the relationship between maternal and fetal hemoglobin versus confounding factors.

Results The prevalence of maternal anemia $(\mathrm{Hb}<11 \mathrm{~g} / \mathrm{dLit})$ before delivery was $24.8 \%(n=150)$ and Hb levels less than $10 \mathrm{~g} / \mathrm{dLit}$ was $6.6 \%$. Overall, $5.8 \%(n=34)$ newborns had a mean $\mathrm{Hb}$ levels of less than $13.7 \mathrm{~g} / \mathrm{dLit}$. A small number of mothers $(3.3 \%, \mathrm{n}=20)$ had preterm delivery and 31 newborn babies (5.3\%) were low birth weight. There was no significant relationship between fetal $\mathrm{Hb}$ and gender, twins, and preterm babies. There was also a positive and significant correlation between maternal and fetal Hb levels ( $\mathrm{r}=0.143, \mathrm{p}<0.001)$.

Conclusion A significant association was observed between fetal as well maternal anemia with mothers' age, preterm birth, duration of iron therapy and occupation. WITH DEFERIPRONE RELATED ARTHROPATHY

doi:10.1136/archdischild-2012-302724.0758
'R Sharma, ${ }^{2} \mathrm{R}$ Anand, 'J Chandra, 'A Seth, 'HK Pemde, 'V Singh. 'Paediatrics, Lady Hardinge Medical College and associated Kalawati Saran Children's Hospital, 2Radiology, Lady Hardinge Medical College, New Delhi, India

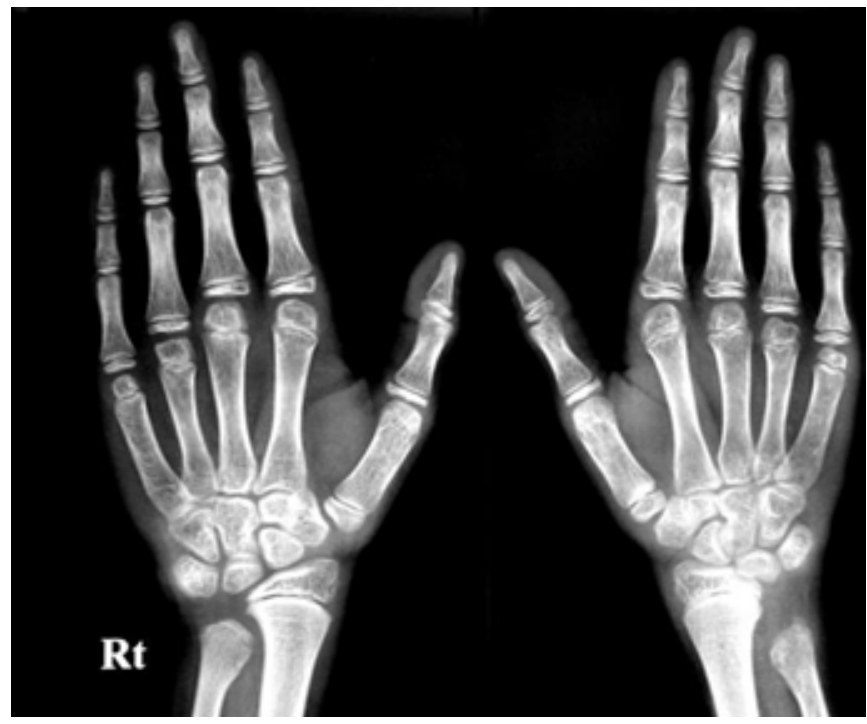

Abstract 758 Figure 1



Abstract 758 Figure 2

Background Regular blood transfusion and iron chelation are the standard of care for thalassemic children. Deferiprone is an effective oral iron chelator but causes significant arthropathy. Though clinical and radiographic features deferiprone related arthropathy have been described, the long-term effects are not known.

Aims To report clinical and radiographic findings in thalassemic children with deferiprone related arthropathy.

Methods Evaluation of routine radiographs of left wrist and hand done for bone age estimation in 40 thalassemic children revealed unique radiographic changes in 13 children with previous or current deferiprone related arthropathy. Subsequently, these children underwent radiographs of both the knee joints.

Results Thirteen patients (10 males: 3 females) aged 10-16 years had abnormal radiographic findings. Median duration of deferiprone 